\title{
The Industrial Indoor Channel: Statistical Analysis of the Power Delay Profile
}

\author{
Emmeric Tanghe, Wout Joseph, member IEEE, Jeffrey De Bruyne, \\ Leen Verloock, and Luc Martens, member IEEE \\ Ghent University / IBBT, Dept. of Information Technology \\ Gaston Crommenlaan 8 box 201, B-9050 Ghent, Belgium \\ Fax: +32 933 14899, E-mail: emmeric.tanghe@intec.UGent.be
}

\section{A. Introduction}

In this paper, an analysis of the wireless channel impulse response in industrial environments is presented. Only limited literature exists which investigates the multipath profile in industrial channels, e.g., [1]. In this work, measurements of the channel impulse response are fitted to the widely used Saleh-Valenzuela model [2]. Measurements have been performed to reflect a specific kind of industrial communication, namely communication between an access point located at considerable height in the factory hall (about $6 \mathrm{~m}$ ) and wireless nodes situated at machine height (about $2 \mathrm{~m}$ ). Motivation for the usefulness of these choices for transmitting and receiving antenna heights is given in [3]. To our knowledge, no model for the industrial channel impulse response for communication between these two heights has yet been reported.

\section{B. Measurement procedure and data pro- cessing}

Propagation measurements were conducted in different production rooms of a wood processing facility located in
Flanders, Belgium. The factory produces and stores wooden flooring products. Raw wooden materials are processed in workplaces by semi-automated machinery used for sawing, breaking, impregnating, and painting the wood. The buildings at the factory site are one-storied, and have a ceiling height of approximately $7 \mathrm{~m}$. All measured industrial halls exhibited similar physical properties: they have concrete floors and metal ceilings supported by steel truss work. Moreover, building walls are made of thick, precast concrete. The industrial inventory at all measurement locations consists for the largest part of similar metal machinery and piping. The machinery extends to heights between 2 and $3 \mathrm{~m}$ above ground level. Fig. 1 presents a view of the measurement environment.

Measurements of the channel impulse reponse were performed in the frequency domain using a Rohde \& Schwarz ZVR vector network analyzer. The frequency of the transmitted signal was swept from $800 \mathrm{MHz}$ to $4 \mathrm{GHz}$ (time domain resolution of $0.31 \mathrm{~ns})$. The wireless channel was probed at 801 points in the measured 


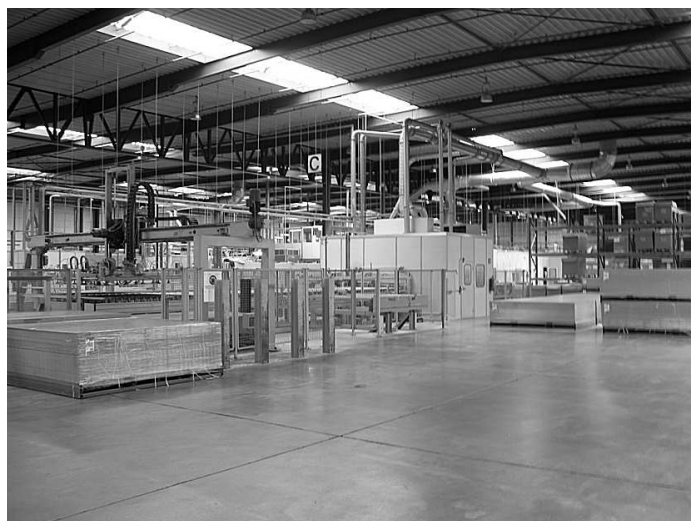

Fig. 1. Measurement environment: production room in a wood processing facility

frequency range (maximum resolvable time delay of $250 \mathrm{~ns}$ ). The swept-frequency signal is amplified by an amplifier of type AR 5S1G4. Omnidirectional, vertically polarized broadband antennas of type European Antennas XPO2V-0.3-10.0/1381 were used for both the transmitting (Tx) and the receiving $(\mathrm{Rx})$ antenna.

The channel impulse response was measured by applying the virtual antenna array method. The Tx was shifted along a uniform linear antenna array (ULA) consisting of 4 elements, and the Rx was moved along a uniform rectangular antenna array (URA) made of $4 \times 3=12$ elements. Thus, the virtual antenna array allows for a total of 48 combinations of Tx and Rx positions. To promote independent fading, the minimum spacing between two array elements was chosen equal to $18.8 \mathrm{~cm}(=$ half a wavelength at $800 \mathrm{MHz}$ ). In total, the virtual antenna array was moved to 10 locations in different industrial halls, and distances of 10 to $35 \mathrm{~m}$ between transmitter and receiver end were covered. Measurements were conducted with Tx and $\mathrm{Rx}$ heights equal to respectively $6 \mathrm{~m}$ and $2 \mathrm{~m}$ above ground level. Fig. 2 shows the measurement setup schematically.

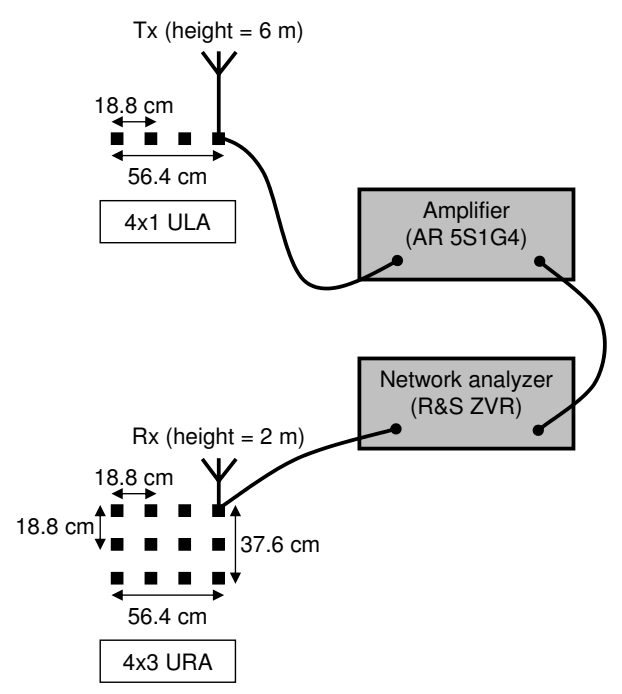

Fig. 2. Measurement setup

For each of the 48 combinations of $\mathrm{Tx}$ and Rx positions in the virtual array, the measured complex channel transfer function is converted to the delay domain using an inverse discrete Fourier transform algorithm, resulting in the complex channel impuls response. Then, the corresponding power delay profile (PDP) is calculated as the squared magnitude of the channel impuls response. Next, the 48 PDPs are linearly averaged to form an averaged power delay profile (APDP).

\section{Modelling}

1) Saleh-Valenzuela model: The main assumption of the Saleh-Valenzuela (SV) model for the APDP is the arrival of multipath components within several distinctly recognizable clusters [2]. In the $\mathrm{S}-\mathrm{V}$ model, the complex channel impulse response $h(\tau)$ as a function of delay $\tau$ is given as: 


$$
h(\tau)=\sum_{l=0}^{\infty} \sum_{k=0}^{\infty} \beta_{k l} e^{j \theta_{k l}} \delta\left(\tau-T_{l}-\tau_{k l}\right) .
$$

In (1), $\beta_{k l}$ and $\theta_{k l}$ are the amplitude gain and phase of the $k$-th ray in the $l$ th cluster, respectively. The parameters $T_{l}$ and $\tau_{k l}$ are the arrival times of $l$-th cluster and the $k$-th ray in the $l$-th cluster, respectively, and $\delta(\cdot)$ denotes the Dirac delta function. In the S-V model, the largescale fading power gain $\overline{\beta_{k l}^{2}}$, i.e. the power gain $\beta_{k l}^{2}$ averaged over an area where large-scale fading remains unchanged, is modeled as a double exponential decay as function of delay:

$$
\overline{\beta_{k l}^{2}}=\overline{\beta_{11}^{2}} e^{-T_{l} / \Gamma} e^{-\tau_{k l} / \gamma}
$$

In (2), $\Gamma$ and $\gamma$ are the cluster and ray power decay constants, respectively. Furthermore, the model assumes that the interarrival times of clusters (i.e., $T_{l}-$ $T_{l-1}$ ) and rays (i.e., $\left.\tau_{k l}-\tau_{(k-1)}\right)$ are exponentially distributed with rate parameters $\Lambda$ and $\lambda$, respectively.

Fig. 3 shows a measured APDP for a separation of $20 \mathrm{~m}$ between the Tx and the Rx. For all measured APDPs, the origin of the delay axis $\tau$ is set to the delay of the first arriving multipath component, and the received power is normalized to the power of the first component.

The onsets $T_{l}$ of individual clusters are determined through visual inspection of the APDP. For example, seven clusters can be identified in Fig. 3, for which the onsets are pointed to by arrows. In the following, the determination of and some novel statistical models for the S$\mathrm{V}$ model parameters are discussed. Due to the limited time resolution of our measurements, the ray arrival rate $\lambda$ could not be determined.

- Ray power decay constant $\gamma$ : For each cluster separately, this parameter is determined by performing linear regression of intra-cluster power in $\mathrm{dB}$ as function of delay (shown as solid straight lines in Fig. 3). In Fig. 3, the decay constant $\gamma$ seems to increase with delay (slope of the solid lines decreases). This is in contrast with the original $\mathrm{S}-\mathrm{V}$ model, wherein $\gamma$ is assumed constant [2]. We propose the following statistical model for $\gamma$ in ns as function of delay $\tau$ in ns:

$$
\ln (\gamma)=1.78+0.02 \cdot \tau+0.76 \cdot Z
$$

In (3), $\ln (\cdot)$ denotes the natural logarithm and $Z$ is a standard normally distributed random variable.

- Cluster power decay constant $\Gamma$ : The $\Gamma$ parameter can be determined through linear regression of cluster peak powers $P_{\text {peak }}$ in $\mathrm{dB}$ and their associated delays $\tau$ in ns (shown as a dashed line in Fig. 3). For all measured APDPs together, the following relationship was obtained:

$$
P_{\text {peak }}=-0.20 \cdot \tau+7.37 \cdot Z
$$

A purely exponential decay of linear peak power as in [2], with decay constant $\Gamma$, would only consider the first term in the right-hand side of (4). However, the random normally distributed second term cannot be easily neglected. Therefore, we propose to model cluster peak power as in (4): an exponential decay of linear power, however, with the inclusion 


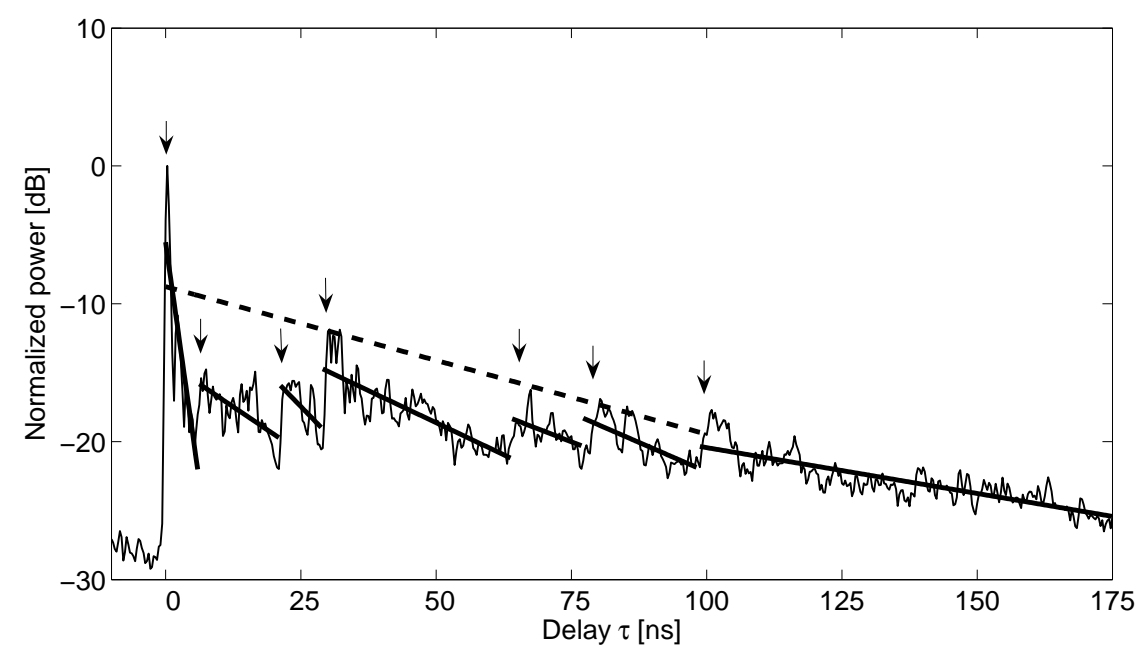

Fig. 3. Averaged power delay profile for a $20 \mathrm{~m}$ measurement

of a statistical error term in order to better fit empirical data.

- Cluster arrival rate $\Lambda$ : The cluster interarrival times $T_{l}-T_{l-1}$ are used to calculate a maximum likelihood estimator of their arrival rate $\Lambda$. For our measurements, the mean cluster interarrival time equals $18.40 \mathrm{~ns}$. A chi-squared goodness-of-fit test retained the null hypothesis that the cluster interarrival times are exponentially distributed at a $5 \%$ significance level. For measurements in industrial environments in [1], mean cluster interarrival times between 13 and $16 \mathrm{~ns}$ are found, which are comparable to our obtained value of $18.40 \mathrm{~ns}$. In literature, when comparing industrial environments to office environments, clusters usually arrive further in between in the latter: mean cluster interarrival times of $60 \mathrm{~ns}$ for measurements in a university building in [4] and 27 to $40 \mathrm{~ns}$ for an office building in [5] were found. This would mean that in office environments, less spa- tially well separated groups of reflective objects (where objects within a group give rise to multipath reflections within the same APDP cluster) are found. Because the inventory in industrial environments (machinery, transport belts, steel piping) is more irregularly shaped, more clusters in rapid succession are expected to be found in these environments. Also, in contrast to office environments, the inventory in industrial environments largely consists of highly reflective metal surfaces [1], [3]. In industrial halls, more objects are therefore expected to actively participate in the wave propagation while giving rise to noticeable received powers at the receiver's end. This means that more clusters could be detected in industrial halls when compared to office buildings.

2) Small-scale fading: In addition to the Saleh-Valenzuela model for the APDP, also the properties of small-scale amplitu- 

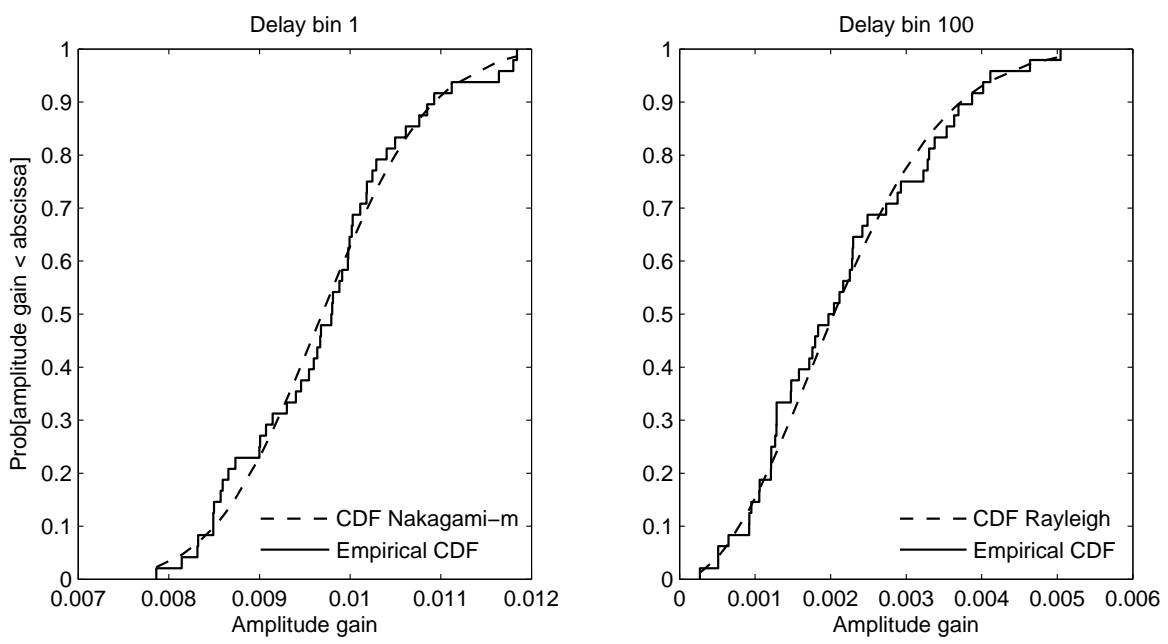

Fig. 4. Empirical CDFs and estimated theoretical CDFs for the amplitude gains in delay bins 1 and 100 of a measurement at $15 \mathrm{~m}$

de fading in the time domain are investigated. For each time delay bin of width $0.31 \mathrm{~ns}$, the most appropriate statistical distribution for the amplitude gains of the 48 small-scale PDPs (used to calculate one large-scale APDP) is selected from a number of candidate distributions (Nakagami-m, Rayleigh, or lognormal) by executing likelihood ratio tests. These tests showed that, in general, Nakagami$m$ fading applies to the amplitude gains in the first delay bin (containing the first-arriving multipath component), while Rayleigh fading seems to be fit for any subsequent delay bins. For the first delay bin of all measured APDPs, estimated values of the $m$ parameter of the Nakagami$m$ fading varied between 3 en 67 . Fig. 4 shows the empirical cumulative distribution functions (CDFs) of amplitude gains in the first and the hundredth delay bin (solid lines) for a measurement at $15 \mathrm{~m} \mathrm{Tx}-\mathrm{Rx}$ separation. These empirical CDFs show good agreement with the estimated theoretical CDFs (dashed lines): Nakagami- $m$ for the first delay bin and Rayleigh for the hundredth delay bin.

\section{REFERENCES}

[1] J. Karedal, S. Wyne, P. Almers, F. Tufvesson, and A. F. Molisch, "A Measurement-Based Statistical Model for Industrial Ultra-Wideband Channels," IEEE Transactions on Wireless Communications, vol. 6, no. 8, pp. 3028-3037, August 2007.

[2] A. A. M. Saleh and R. A. Valenzuela, "A Statistical Model for Indoor Multipath Propagation," IEEE Journal on Selected Areas in Communications, vol. 5, no. 2, pp. 128-137, February 1987.

[3] E. Tanghe, W. Joseph, L. Verloock, L. Martens, H. Capoen, K. Van Herwegen, and W. Vantomme, "The Industrial Indoor Channel: Large-Scale and Temporal Fading at 900, 2400, and $5200 \mathrm{MHz}$," IEEE Transactions on Wireless Communications, vol. 7, no. 7, pp. 2740-2751, July 2008.

[4] I. Marinović, I. Zanchi, and Z. Blažević, "Estimation of Channel Parameters for "Saleh-Valenzuela" Model Simulation," in Proceedings of the International Conference on Applied Electromagnetics and Communications, Dubrovnik, HR, October 2005, pp. 1-4.

[5] P. Pagani and P. Pajusco, "Experimental Analysis of the Ultra Wideband Propagation Channel over the $3.1 \mathrm{GHz}-10.6 \mathrm{GHz}$ Frequency Band," in Proceedings of the IEEE International Symposium on Personal, Indoor, and Mobile Radio Communications, Helsinki, FI, September 2006, pp. 1-5. 
15th Annual Symposium of the IEEE/CVT Benelux Chapter

Invitation 13 november 2008

It is our pleasure to invite you to the 15th Symposium on Communications and Vehicular Technology in the Benelux SCVT 2008. Established in 1993, the Symposium is an annual event organized by the IEEE chapter on Communications and Vehicular Technology. The Symposium is aimed at presenting and discussing the latest scientific and technical advances in communication systems and vehicular communication technology.

Keynote address

- "Software Defined Radio, flexibility for low power?" by Filip Louagie, IMEC

- "Exploiting cooperation in wireless networks" by Jorge Garcia Vidal, UPC

Sponsors

- IEEE

"Read more

๑

Proceedings available on USB stick 